\title{
Respostas a aplicação de concentrações de prohexadione cálcio em amoreira-preta (Rubus spp.) cv. Tupy
}

Elói Evandro Delazeri', Andressa Vighi Schiavon², Luiz Antônio Camacho Nardello', Cristiano Geremias Hellwig ${ }^{2}$, Luis Eduardo Corrêa Antunes ${ }^{3}$

\footnotetext{
' Engenheiro Agrônomo, Mestrando no Programa de Pós-Graduação em Agronomia na área de Fruticultura de Clima Temperado, Universidade Federal de Pelotas (UFPel), Pelotas-RS.

E-mail: eloidelazeri@gmail.com*, luiz.nardello@gmail.com.

${ }^{2}$ Engenheiro Agrônomo, Mestre em Agronomia, Doutorando no Programa de Pós-Graduação em Agronomia na área de Fruticultura de Clima Temperado, Universidade Federal de Pelotas (UFPel), Pelotas-RS.

E-mail: andressa.vighi@gmail.com, cristiano.hellwig@gmail.com.

${ }^{3}$ Engenheiro Agrônomo, Doutor em Agronomia, Pesquisador Embrapa Clima Temperado-Pelotas-RS.

E-mail: luis.antunes@embrapa.br.
}

\section{Resumo}

O objetivo deste trabalho foi avaliar os efeitos da aplicação de diferentes concentrações de prohexadione cálcio na prorrogação do período de colheita e nos atributos físico-químicos de frutas de amoreira-preta, cultivar Tupy. Os tratamentos foram constituídos de quatro concentrações de prohexadione cálcio (PCa) (100; 200; 400; 800 mg. $\left.\mathrm{L}^{-1}\right)$, além de um tratamento testemunha sem aplicação. Foram avaliados os dias decorridos após a aplicação (DAP), massa média das frutas, sólidos solúveis, acidez titulável e cor: luminosidade ( $\left.\mathrm{L}^{*}\right)$, ângulo hue ( $\left.{ }^{\circ} \mathrm{Hue}\right)$, cromaticidade ( $\left.C^{*}\right)$. A PCa estende o período de colheitas das frutas de amora-preta em decorrência do aumento das concentrações aplicadas. $\mathrm{O}$ aumento das concentrações de $\mathrm{PC}$ a diminuem a massa média de frutas e aumentam o teor de sólidos solúveis, já para os parâmetros de cor, o aumento das concentrações aplicadas as plantas de amoreira-preta, resultam em frutas com cor vermelha mais intensa ( ${ }^{\circ} \mathrm{Hue}$ ), com aumento dos valores de luminosidade $\left(L^{*}\right)$ e intensidade da cor (C*).

Palavras chaves: Reguladores de crescimento. Produção extemporânea. Qualidade físico-química.

\section{Abstract}

\section{Responses to application of prohexadione calcium concentrations in blackberry (Rubus spp.) cv. Tupy}

This work aimed to evaluate the effects of applying different prohexadione calcium concentrations on the extension of harvest period and on physicochemical attributes of blackberry fruits cv. Tupy. The treatments consisted of four concentrations of prohexadione calcium (PCa) (I00; 200; 400; 800 mg. $\mathrm{L}^{-1}$ ), as well as a control treatment without application. Were evaluated: days after application (DAP), average fruit mass, soluble solids, titratable acidity and color: luminosity $\left(\mathrm{L}^{*}\right)$, hue angle ( $\left.{ }^{\circ} \mathrm{Hue}\right)$, chromaticity $\left(\mathrm{C}^{*}\right)$. The PCa extends harvest period of blackberry cv. Tupy fruits due to the increase of applied concentrations. Increasing PCa concentrations decrease the average fruit mass and increase the soluble solids content, while for the color parameters, the increased concentrations applied to blackberry plants result in more intense red color ( ${ }^{\circ} \mathrm{Hue}$ ), with increasing brightness $(\mathrm{L} *)$ and color intensity $(\mathrm{C} *)$ values.

Keywords: Growth regulators. Extemporaneous production. Physicochemical quality. 


\section{Introdução}

A área cultivada com amoreira-preta vem crescendo gradativamente nos últimos anos em diversas regiões do mundo, principalmente Estados unidos e Europa (CLARK; FINN, 20I4). Este aumento na demanda pela fruta é atribuído a diversos fatores, desde econômicos a sociais, e, principalmente, pelas suas elevadas qualidades fitoquímicas e nutracêuticas, que trazem diversos benefícios à saúde do consumidor. A amoreira-preta tem despertado o interesse dos produtores, devido ao baixo custo de produção, facilidade de manejo, rusticidade e a baixa demanda na utilização de defensivos agrícolas (ANTUNES et al., 20l4).

O principal entrave da cultura, no tocante ao período produtivo, é a concentração da safra, que ocorre de novembro a janeiro no sul do Brasil, ocasionando escassez nos demais meses do ano (ANTUNES; RASEIRA, 2004). Este fator é o principal limitante no manejo da produção e comercialização, destinando grande parte do quantitativo colhido para o processamento industrial, diminuindo a oferta do produto in natura ao consumidor e o retorno financeiro ao produtor.

A busca por técnicas que alterem o período produtivo envolve a aplicação de podas extemporâneas e reguladores de crescimento. As podas extemporâneas podem ser realizadas, por exemplo, de forma escalonada, tanto no verão quanto no inverno. Quando realizadas no verão, estimulam a brotação de novos ramos, onde as gemas podem se diferenciar e florescer de forma antecipada (ANTUNES et al., 2006). A realização das podas no inverno (final de julho e início de agosto) tende a diminuir a produtividade, porém, pode ocasionar produção fora de época, sendo possível obter, muitas vezes, maior retorno financeiro pelo produto comercializado (CAMPAGNOLO; PIO, 20I2).

A utilização de reguladores vegetais pode ser uma alternativa para a produção extemporânea da amoreira-preta. Dentre eles se destacam os inibidores da biossíntese de giberelinas, utilizados principalmente para a redução de crescimento dos ramos e atraso na maturação das frutas. Os principais inibidores da biossíntese de giberelinas são: paclobutrazol (PBZ), prohexadione cálcio (PCa), etil trinexapac e cloreto de chlormequat (HAWERROTH et al., 20I2; RUFATTO et al., 20I4; SILVEIRA, 20I5).

Diante do exposto, objetivou-se com este trabalho avaliar os efeitos de concentrações de prohexadione cálcio sobre a maturação e atributos físico-químicos de frutas de amoreira preta.

\section{Material e Métodos}

O experimento foi conduzido durante a safra 2018/2019, na área experimental pertencente à Embrapa Clima Temperado-Pelotas/RS, latitude de 31 ${ }^{\circ} 40^{\prime}$ sul e longitude 52²6' oeste, com $60 \mathrm{~m}$ de altitude. $\mathrm{O}$ clima da região, segundo Koppen, é do tipo "Cfa" - temperado úmido com verões quentes, temperatura do ar e precipitação pluviométrica média anual de $17,9^{\circ} \mathrm{C}$ e $1500 \mathrm{~mm}$, respectivamente.

As plantas utilizadas possuíam três anos de idade, todas da cultivar Tupy, com espaçamento de 0,60 m entre plantas e 3,0 m entre linhas (5.555 plantas. ha ${ }^{-1}$ ). O sistema de condução adotado foi em espaldeira, com arrames espaçados em $0,5 \mathrm{~m}$, até a altura de $2,0 \mathrm{~m}$. O delineamento experimental foi em blocos ao acaso, composto de cinco tratamentos, com quatro repetições. Cada unidade experimental foi composta por cinco plantas com cinco hastes cada, as quais foram podadas e padronizadas com altura de $80 \mathrm{~cm}$ da superfície do solo. A poda de verão foi realizada no dia II/02/20 I8 e a poda de condução realizada no dia 15/08/20I8.

Os tratamentos foram constituídos de quatro concentrações de PCa (I00; 200; 400; 800 mg. $\left.\mathrm{L}^{-1}\right)$, juntamente com um tratamento testemunha sem aplicação. O produto comercial utilizado foi o Viviful ${ }^{\circledR}$, contendo $27,5 \%$ de ingrediente ativo. A aplicação dos tratamentos foi feita por aspersão através de pulverizador costal, utilizando-se volume de calda correspondente a I.000 L ha-1, sendo esta realizada em 27/09/20 I8, quando todas as plantas estavam em plena brotação.

Para os valores referentes ao atraso da maturação das frutas, foram contabilizados os dias decorridos a partir da aplicação das concentrações de PCa até a data da última colheita de frutas comercialmente viáveis de cada parcela, obtendo-se a variável dias após a aplicação (DAP). A massa média das frutas em cada parcela foi calculada através do quociente entre a massa e o número de frutas obtidas em cada colheita, sendo considerados para pesagem apenas as frutas sem danos físicos e fisiológicos.

Os sólidos solúveis (SS) foram determinados por refratometria, através de refratômetro digital portátil de bancada, com correção da temperatura e os resultados expressos em ${ }^{\circ}$ Brix. A acidez titulável total (ATT) foi determinada através da titulação potenciométrica com $\mathrm{NaOH} 0, \mathrm{IN}$ até $\mathrm{pH} 8, \mathrm{I}$ de $10 \mathrm{~g}$ da amostra tritu- 
rada e diluída em $90 \mathrm{ml}$ água destilada e os resultados são expressos em \% de ácido cítrico. $\mathrm{O}$ pH foi determinado com peagâmetro digital (marca e modelo) diretamente no suco das frutas com o uso de um medidor de $\mathrm{pH}$, com correção automática de temperatura.

A coloração da epiderme das frutas foi medida com o auxílio de colorímetro eletrônico (Minolta 300), dotado de fonte de luz D65, com abertura de $8 \mathrm{~mm}$ de diâmetro, calibrado segundo orientação do fabricante. Este aparelho efetua a leitura da cor em escala tridimensional L*, a* e b* ou CIELAB, onde os valores de $L^{*}$ correspondem à luminosidade ou claridade e variam de 100 (branco) a zero (preto). As coordenadas a* e b* indicam a direção da cor: -a* é a direção do verde e $+a^{*}$ a direção do vermelho; -b* é a direção do azul e $+b^{*}$ a direção do amarelo. A partir destes valores, são calculados os valores da tonalidade da cor (ângulo 'hue). $\mathrm{O}^{\circ}$ Hue possui variação de: 0 a $12^{\circ}$ para a coloração vermelha, 13 a $41^{\circ}$ para a coloração alaranjada, 42 a $69^{\circ}$ para a coloração amarelo, 70 a $166^{\circ}$ para verde, 167 a $25 I^{\circ}$ para azul, 252 a $305^{\circ}$ para violeta e 306 a $359^{\circ}$ para vermelho, perfazendo $360^{\circ}$. A fórmula I expressa o referido cálculo.

$$
\text { oHue }=\left(\operatorname{Tan}^{-1} \mathrm{~b}^{*} / \mathrm{a}^{*}\right)
$$

O croma indica a intensidade ou a saturação de cor, sendo definido pela distância do ângulo hue do centro do diagrama tridimensional. A fórmula 2 expressa o referido cálculo.

$$
\text { Croma }=\left(a^{* 2}+b^{* 2}\right)^{1 / 2}
$$

As variáveis estudadas foram analisadas utilizando-se o programa de análises estatísticas Sisvar 5.6 (Ferreira, 2014), desenvolvido pela Universidade Federal de Lavras-UFLA. Para analisar os efeitos das diferentes concentrações de $\mathrm{PCa}$, os dados foram submetidos a análise de variância e a regressão polinomial ao nível de $5 \%$ de significância.

\section{Resultados e Discussões}

Para os valores referentes ao período de colheita das frutas, houve variação significativa entre as diferentes concentrações aplicadas de PCa (Figura I A), onde em comparação com o tratamento controle, a maior concentração $\left(800 \mathrm{mg} \mathrm{L}^{-1}\right)$ prolongou o período de colheita em 17 dias, demonstrando que a PCa interfere na maturação das frutas da amoreira-preta.

O atraso na maturação das frutas possivelmente está associado à aplicação de PCa, pois esta interfere na biossíntese de giberelinas, antocianinas e flavonoides, sendo também responsável por atuar na inibição da produção de etileno. Esta inibição ocorre pelo bloqueio da enzima ACC-oxidase, decorrente da semelhança entre a estrutura molecular da PCa e o ácido ascórbico, o qual é co-substrato da enzima ACC-oxidase (RADEMACHER, 20 I4). A enzima ACC-oxidase é responsável por catalisar o ACC (ácido I-carboxílico-I-aminociclopropano) em sua última etapa antes de ser convertido em etileno no ciclo de Yang (TAIZ et al., 20I7), razão pela qual pode-se explicar a diminuição na velocidade de maturação das frutas de amora-preta.

A atuação do etileno ocorre principalmente sobre a parede celular das frutas, degradando as pectinas, as quais são as principais constituintes da lamela média. Esses e outros processos são desencadeados por tal hormônio, que exerce um importante papel durante o amadurecimento das frutas. $O$ etileno desencadeia ainda diversos processos, tais como desenvolvimento da cor, perda de firmeza e formação do aroma (BALBONTÍN et al., 2007). Atrasos na maturação de frutas foram verificados em diversas culturas onde foi aplicada a PCa, a exemplo de pereiras (HAWERROTH et al., 20I2), videiras (LO GIUDICE; WOLF; ZOECKLEIN, 2004; RUFATTO et al., 20I4) e macieiras (SILVEIRA, 20I5), o que ratifica os resultados encontrados neste trabalho. Estes resultados indicam que o efeito deste fitorregulador depende da espécie, da concentração, e, provavelmente, do estádio fenológico em que ele é aplicado.

Para os valores de massa média de frutas, a aplicação de diferentes concentrações de PCa em amoreiras cv. Tupy interferiu significativamente no peso médio de frutas (Figura I B), demonstrando que as concentrações mais elevadas (400 e $800 \mathrm{mg} \mathrm{L}^{-1}$ ) tendem a diminuir a massa média de frutas em 22,7 e 18,3\%, respectivamente, afetando negativamente a produtividade. A PCa inibe as etapas finais da biossíntese de giberelinas por interferir na $3 \beta$-hidroxilação, diminuindo o desenvolvimento vegetativo devido à redução 
Figura I

Efeito da aplicação de prohexadione cálcio no período de maturação $(A)$, massa média (B), teor de sólidos solúveis $(C)$ e ATT (D) em frutos de amoreira preta cv. Tupy.

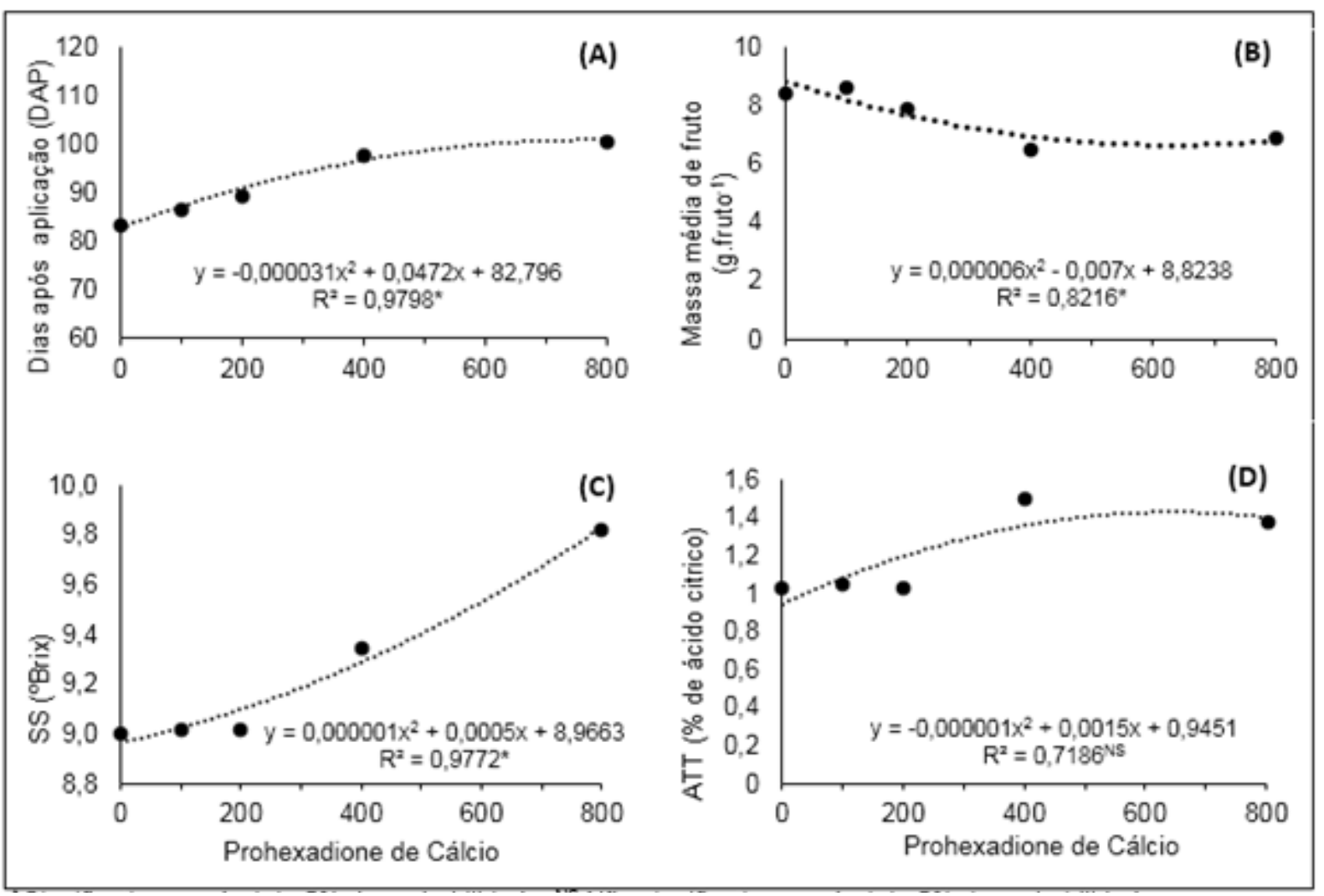

* Significativo ao nível de $5 \%$ de probabilidade. NS Não significativo ao nível de $5 \%$ de probabilidade. Fonte: Autores (2019).

dos níveis endógenos de giberelinas biologicamente ativas $\left(\mathrm{GA}_{1}\right)$ e acumulando seu precursor biologicamente inativo $\left(\mathrm{GA}_{20}\right)$. Desta forma, ocorre um menor alongamento celular, justificando o menor crescimento e peso das frutas da amoreira-preta (RADEMACHER et al., 2006).

Os resultados encontrados neste experimento corroboram com o exposto por Ozbay e Ergun (20I5), na cultura da berinjela, onde demonstraram uma redução de $7 \%$ na massa média das frutas após a aplicação de PCa. Sugar, Elfving e Mielke (2004) descrevem que, além da diminuição da massa média de frutas, a PCa provoca diminuição no retorno da floração em plantas de pereira cv. Bosc.

A resposta a uma única aplicação de PCa mantém seu efeito por aproximadamente 3 a 4 semanas, sendo que a concentração a ser aplicada depende do vigor de crescimento estimado da planta e este, por sua vez, depende de vários fatores como: variedade, idade da planta, capacidade de retenção e pegamento das frutas e características vinculadas ao solo (localização geográfica, disponibilidade de nutrientes, água...). Várias aplicações de PCa em baixas concentrações são mais efetivas em inibir o crescimento das brotações quando comparadas a uma única aplicação de alta concentração, pois este regulador de crescimento apresenta efeito temporário, de três a quatro semanas (UNRATH, 1997).

O conteúdo de sólidos solúveis apresentou um crescimento significativo com o aumento das concentrações de PCa (Figura I C). Concentrações crescentes resultam em crescimento exponencial do teor de sólidos solúveis nas frutas de amoreira-preta, estando relacionado ao menor tamanho da fruta, - que ocasiona uma maior concentração de sólidos solúveis nos mesmos. Diversos são os trabalhos realizados com aplicação de PCa em frutíferas, porém, resultados referentes ao teor de sólidos solúveis (SS) são distintos aos encontrados no presente trabalho. Atay e Kyoncu (2017), ao avaliarem aplicações de PCa durante três anos em macieiras da cultivar Golden Delicious, não encontraram mudanças nos teores de sólidos solúveis, e, em pereiras da cultivar D’Anjou; Einhorn, Pasa e Turner (20I4) demonstraram diminuição nos valores de SS. Estes resultados demonstram que existe variabilidade nas respostas a aplicação de PCa para as diferentes espécies. 
Poucas informações são encontradas na literatura sobre a aplicação de PCa em pequenas frutas, e os valores de SS encontrados neste trabalho podem estar atrelados ao tamanho médio da fruta, que, segundo Medjdoub, Val e Blanco (2004) relacionam o aumento da quantidade de sólidos solúveis com a quantidade de água na fruta. Desta forma, quanto maior a fruta, maior a quantidade de água e mais dissolvidos estão os açúcares e os sólidos solúveis, ocorrendo o inverso com a diminuição do tamanho do mesmo.

A variável acidez titulável (AT) (Figura I D) não apresentou diferença significativa entre as concentrações avaliadas de PCa. Os valores encontrados variam de I,03 (testemunha) a I,38 mg de ácido cítrico $100 \mathrm{~g}^{-1}$ de amostra (dose $800 \mathrm{mg}^{\mathrm{L}^{-1}}$ ). Estes valores são inferiores aos encontrados por Hirsch et al. (20l2) na região de Pelotas/RS, os quais obtiveram variação de I,49 a I,63 mg de ácido cítrico $100 \mathrm{~g}^{-1}$ de amostra. Segundo Segantini et al. (20l4), os valores da acidez titulável nas frutas de amoreira-preta podem ser alterados por influência de diversos fatores, como tipo de solo, época de desenvolvimento das frutas e adubação, fatores estes que podem explicar a disparidade com os valores encontrados no presente trabalho.

Referente ao potencial hidrogeniônico $(\mathrm{pH})$ das frutas de amoreira-preta tratadas com PCa (Figura 2 A), estas apresentaram valores não significativos. Os valores observados neste experimento variam de 3,00 (testemunha) e 2,87 (dose $800 \mathrm{mg} \mathrm{L}^{-1}$ ). Hirsch et al. (2012) avaliando compostos fitoquímicos em diferentes cultivares de amora, encontraram valores de $\mathrm{pH}$ entre 2,90 e 3,22 para a cultivar Tupy, sendo estes valores esperados para frutas ácidas a doce-ácidas como a amora-preta.

Para a variável luminosidade $\left(L^{*}\right)$, observaram-se valores significativos para as diferentes doses da PCa (Figura 2 B), indicando que o aumento das concentrações diminuem a intensidade da cor das frutas da amoreira-preta. Para os valores de L*, quanto mais próximos de zero mais intensa é a cor da fruta, sendo que para a amoreira, estes valores tendem a diminuir com o amadurecimento das frutas, indicando que a cor se torna mais intensa ou escura (TOSUN; USTUN; TEKGULER, 2008).

A amora-preta possui elevado teor de antocianinas e baixo teor de compostos fenólicos e flavonoides (FERREIRA; ROSSO; MERCADANTE, 2010). A diminuição da síntese destes compostos, principalmente das antocianinas, acarretam na diminuição da cor das frutas da amoreira e, consequentemente, o atraso na colheita dos mesmos. A coloração das frutas é um importante parâmetro para produtores e consumidores, sendo que esta indica as condições ideais para a colheita e comercialização, o ponto de colheita das frutas da amoreira é determinado quando o mesmo encontra-se totalmente pretos (visualmente).

Os valores atribuídos ao ${ }^{\circ}$ Hue $(\mathrm{H})$ (Figura $2 \mathrm{C}$ ) não se alteraram significativamente, porém, percebe-se a diminuição dos valores aferidos com o aumento das concentrações de PCa aplicadas as plantas de amoreira preta. Os valores de $\mathrm{H}$ obtidos tendem ao vermelho, e, juntamente com o aumento dos valores de luminosidade ( $\left.\mathrm{L}^{*}\right)$, demonstram que no momento da colheita das frutas, visualmente não é possível distinguir tal variação na luminosidade e na cor das frutas, sendo que estes estavam aptos para a colheita. Tais resultados demonstram que o processo de formação de cor sofre influência das concentrações de PCa aplicadas.

A PCa está envolvida no metabolismo dos flavonoides e em altas concentrações inibe a formação de antocianinas nas flores e em outros órgãos da planta, proporcionando frutas com menor intensidade de cor (RADEMACHER, 2000). O mesmo autor descreve que a PCa é estruturalmente semelhante ao ácido 2-oxaloglutárico, permitindo que o composto iniba as dioxigenases dependentes do 2-oxoglutarato, os quais estão envolvidos na formação de giberelinas ativas responsáveis pela síntese e metabolismo de antocianinas. Zhang e Whiting (20I I) aplicando PCa isoladamente e PCa associado a ácido giberélico em cerejas cultivar Bing, observaram atrasos na maturação e frutas com coloração inferior aos do tratamento controle, corroborando com os resultados do presente trabalho.

Os valores de Croma ( $\mathrm{C}^{*}$ ) obtiveram comportamento significativo, demonstrando que o aumento das concentrações de PCa aplicadas as plantas de amoreira preta tendem a intensificar suas cores (Figura $2 \mathrm{D}$ ). Os valores atribuídos ao C* expressam a saturação em termos de pigmentos de cor, sendo que, valores próximos de 0 (zero) indicam cores neutras (cinza) e valores próximos a 60 para cores mais intensas ou vívidas (SILVA et al., 20I I). Neste caso, o aumento das concentrações de PCa aplicadas, tendem a produzir frutas com coloração vermelha mais intensa do que o tratamento controle. Tais valores divergem dos encontrados por Hirsch et al. (20I2) para a cultivar Tupy, sendo que estes encontraram valores médios de 19,2, nas condições de Pelotas/RS. Tal divergência pode estar relacionada a época de desenvolvimento das frutas e colheita, adubação e tipo de solo (SEGANTINI et al., 20I4). 
Figura 22

Efeito da aplicação de prohexadione cálcio sobre o $\mathrm{pH}(\mathrm{A})$, liminosidade $\left(\mathrm{L}^{*}\right)(\mathrm{B})$, oHue $(C)$

e cromaticidade (C*) (D) em frutos de amoreira preta cv. Tupy.

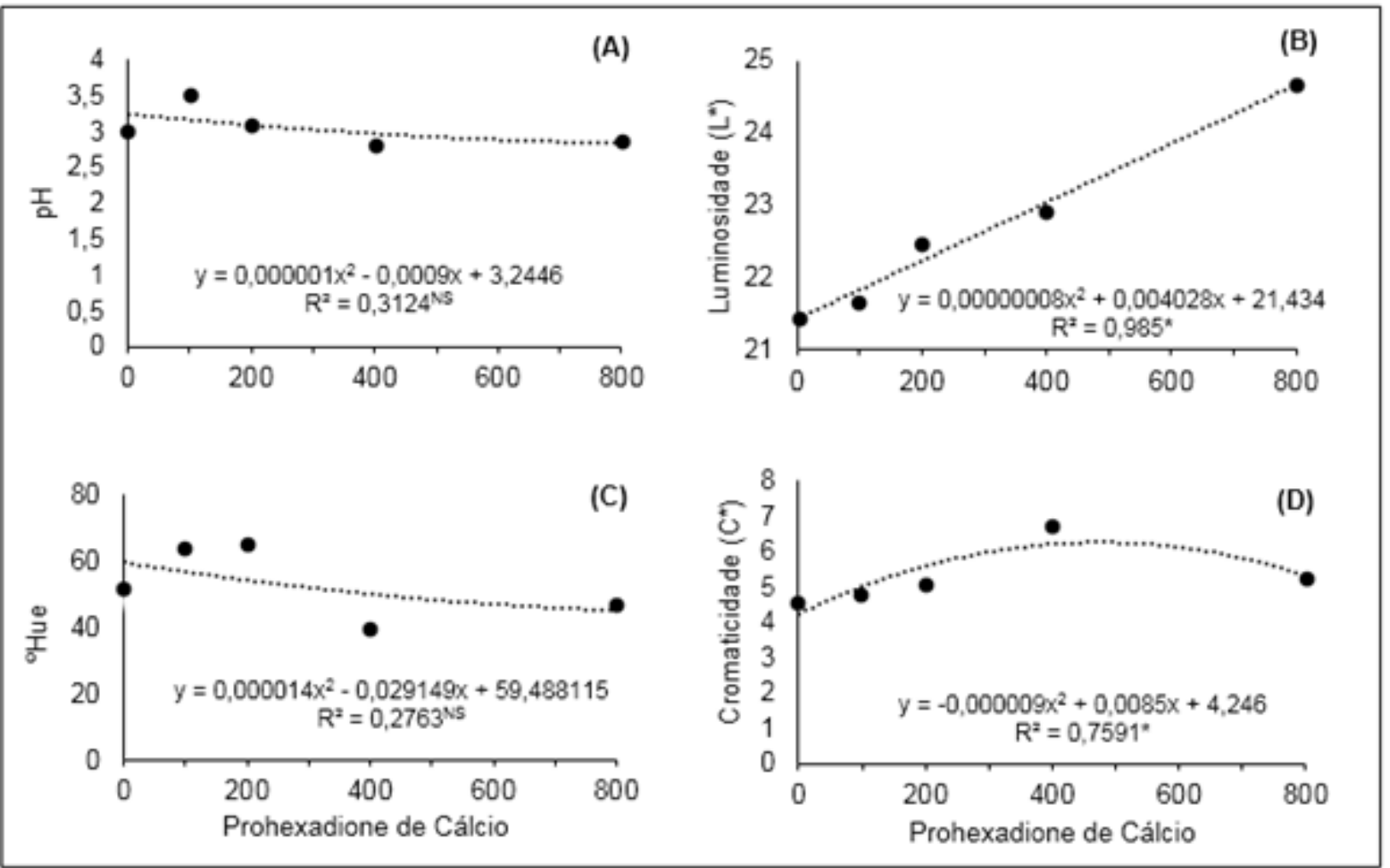

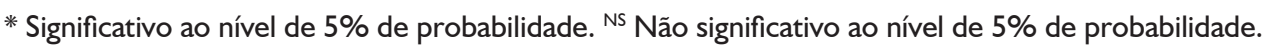
Fonte: Autores (2019).

\section{Conclusões}

A utilização de prohexadione cálcio retarda o processo de formação de cor e maturação das frutas de amoreira-preta, estendendo o período de colheita.

A massa média das frutas é afetada pelo aumento das concentrações de PCa, diminuindo a massa média de fruta e aumentando a concentração de sólidos solúveis.

\section{Agradecimentos}

À Embrapa Clima Temperado, Coordenação de Aperfeiçoamento de Pessoal de Nível Superior (CAPES) e ao Conselho Nacional de Desenvolvimento Cientifico e Tecnológico (CNPq) pelo fomento à pesquisa e financiamento de bolsas de estudo.

\section{Referências}

ANTUNES, L. E. C.; RASEIRA, M. C. B. Aspectos técnicos da cultura da amora-preta. Pelotas: Embrapa-CPACT, 2004. 54p. (Embrapa, documentos I22). Disponível em: https://www.embrapa.br/ en/clima-temperado/ busca-de-publicacoes//publicacao/7448/2/aspectos-tecnicos-da-cultura-da-amora-preta. Acesso em: 16 jun. 2019.

ANTUNES, L. E. C.; TREVISAN, R.; GONÇALVES, E. D.; FRANZON, R. C. Produção extemporânea de amora-preta. Revista Brasileira de Fruticultura, Jaboticabal, v. 28, n. 3, p. 430-434, 2006.

ANTUNES, L. E. C.; PEREIRA, I. S.; PICOLOTTO, L.; VIGNOLO, G. K.; GONÇALVES, M. A. Produção de amoreira-preta no Brasil. Revista Brasileira de Fruticultura, Jaboticabal, v. 36, n. I, p. 100-III, 2014.

ATAY, A. N.; KOYONCU, F. Impact of repeated yearly applications of prohexadione-calcium on vegetative and reproductive Growth of 'golden delicious'/m.9 apple trees. Journal of Horticultural Research, Skierniewice, vol. 25, n. I, p. 47-54, 2017. 
BALBONTÍN, C.; EASTMAN, C. G.; VERGARA, M.; HERRERA, R.; LEÓN, M. A. M. Treatment with I-MCP and the role of ethylene in aroma development of mountain papaya fruit. Postharvest Biology and Technology, Amsterdam, v. 43, n. I, p. 67-77, 2007.

CAMPAGNOLO, M. A.; PIO, R. Produção da amoreira-preta ‘Tupy’ sob diferentes épocas de poda. Ciência Rural, Santa Maria, v. 42, n. 2, 2012.

CLARK, J. R.; FINN, C. E. Cultura da amora-preta no mundo. Revista Brasileira de Fruticultura. Jaboticabal, v.36, n.I, pp.46-57, 2014.

EINHORN, T. C.; PASA, M. S.; TURNER, J. 'D’Anjou’ Pear Shoot Growth and Return Bloom, but Not Fruit Size, Are Reduced by Prohexadione-Calcium. HortScience, [Alexandria], v. 49, n. 2, p. I80-I87, fev. 2014.

FERREIRA, Daniel Furtado. Sisvar: a Guide for its Bootstrap procedures in multiple comparisons. Ciência e Agrotecnologia, Lavras, v. 38, n. 2, 2014.

FERREIRA, D. S.; ROSSO, V. V. de; MERCADANTE, A. Z. Compostos bioativos presentes em amora-preta (Rubus spp.). Revista Brasileira de Fruticultura, Jaboticabal, v. 32, n. 3, p. 664-674, Sept. 2010.

HAWERROTH, F. J.; PETRI, J. L.; FACHINELlO, J. C.; HERTER, F. G.; PREZOTTO, M. E.; HASS, L. B.; PRETTO, A. Redução da poda hibernal e aumento da produção de pereiras 'Hosui' pelo uso de prohexadiona cálcio. Pesquisa Agropecuária Brasileira, Brasília, v. 47, n. 7, p. 939-947, jul. 2012.

HIRSCH, G. E.; FACCO, E. M. P.; RODRIGUES, D. B.; VIZZOTO, M.; EMANUELLI, T. Caracterização físico-química de variedades de amora-preta da região sul do Brasil. Ciência Rural, Santa Maria, v. 42, n. 5, 2012.

LO GIUDICE, D.; WOLF, T. K.; ZOECKLEIN, B. W. Effects of prohexadione-calcium on grape yield components and fruit and wine composition. American Journal of Enology and Viticulture, v. 55, n. I, p. 73-83, 2004.

MEDJDOUB, R.; VAL, J.; BLANCO, A. Prohexadione-Ca inhibits vegetative growth of 'Smoothee Golden Delicious' apple trees. Scientia Horticulturae, [Amsterdam], v. I0 I, n. 3, p. 243-253, 2004.

OZBAY, N; ERGUN, N. Prohexadione calcium on the growth and quality of eggplant seedlings. Pesquisa Agropecuaria Brasileira, Brasília, v. 50, n. 10, p.932-938, out. 2015.

RADEMACHER, W. Growth retardants: effects on gibberellin biosynthesis and other metabolic pathways. Annual Review of Plant Physiology and Plant Molecular Biology, Palo Alto, v. 5 I, n. I, p. 50 I-53 I, 2000.

RADEMACHER, W. Prohexadione-ca and trinexapac-ethyl: similarities in structure but differences in biological action. Acta Horticulturae, v. 1042, p. 33-4I, jul. 2014.

RADEMACHER, W.; SPINELLI, F; COSTA, G. Prohexadione-Ca: Modes of action of a multifunctional plant bioregulator for fruit trees. Acta Horticulturae, v. 727, p. 97-106, 2006.

RUFATTO, L.; BRIGHENTI, A. F.; MACEDO, T. A.; BEM, B. P.; ALLEBRANDT, R.; SOUZA, D. S.; BRUNA, D. D., MACRON FILHO, J. L. Effects of prohexadione-calcium on yield components and fruit composition of cabernet sauvignon in southern Brazil. In: WORLD CONGRESS OF VINE AND WINE. 37., 20I4. Anais... Argentina, 2014.

SEGANTINI, D. M.; LEONEL, S.; CUNHA, A. R. da; FERRAZ, R. A.; RIPARDO, A. K. da S. Exigência térmica e produtividade da amoreira-preta em função das épocas de poda. Revista Brasileira de Fruticultura, Jaboticabal, v. 36, n. 3, p. 568-575, Sept. 2014.

SILVA, M. C. da; ATARASSI, M. E.; FERREIRA, M. D.; MOSCA, M. A. Qualidade pós-colheita de caqui 'Fuyu' com utilização de diferentes concentrações de cobertura comestível. Ciência e Agrotecnologia, v. 35, n. I, p. I44- I5 I, 20 I I.

SILVEIRA, J. P. G., Qualidade e distúrbios fisiológicos de maçãs em resposta a giberelinas e prohexadiona-cálcio. 20I5. Tese (Doutorado em Produção Vegetal). Universidade do Estado de Santa Catarina, Lages, 20I5.

SUGAR, D.; ELFVING, D. MIELKE, E. A. Effects of prohexadione-calcium on fruit size and return bloom in pear. HortScience, [Alexandria], v. 39, n. 6, p.I305-I308, 2004.

TAIZ, L.; ZEIGER, E.; MOLLER, I.; MURPHY, A. Fisiologia e desenvolvimento vegetal. 6 ed. Porto Alegre: Artmed, 20 I 7.

TOSUN, I.; USTUN, N. S; TEKGULER, B. Alterações físicas e químicas durante o amadurecimento de frutos de amora-preta. Scientia Agrícola, Piracicaba, v. 65, n. I, p. 87-90, fev. 2008.

UNRATH, C. R. Prohexadione calcium: A promising chemical for controlling vegetative growth of apples. HortScience, [Alexandria], v. 34, n. 7, p. 1014-1018, Dec. 1997.

ZHANG, C; WHITING, M. Pre-harvest foliar application of Prohexadione-Ca and gibberellins modify canopy sourcesink relations and improve quality and shelf life of 'Bing' sweet cherry. Plant Growth Regulation, v. 65, n. I, p. I45I56, 26 mar. 201 I. 